\title{
A Functional-Based Segmentation of Human Body Scans in Arbitrary Postures
}

\author{
Naoufel Werghi, Member, IEEE, Yijun Xiao, and Jan Paul Siebert, Member, IEEE
}

\begin{abstract}
This paper presents a general framework that aims to address the task of segmenting three-dimensional (3-D) scan data representing the human form into subsets which correspond to functional human body parts. Such a task is challenging due to the articulated and deformable nature of the human body. A salient feature of this framework is that it is able to cope with various body postures and is in addition robust to noise, holes, irregular sampling and rigid transformations. Although whole human body scanners are now capable of routinely capturing the shape of the whole body in machine readable format, they have not yet realized their potential to provide automatic extraction of key body measurements. Automated production of anthropometric databases is a prerequisite to satisfying the needs of certain industrial sectors (e.g., the clothing industry). This implies that in order to extract specific measurements of interest, whole body 3-D scan data must be segmented by machine into subsets corresponding to functional human body parts. However, previously reported attempts at automating the segmentation process suffer from various limitations, such as being restricted to a standard specific posture and being vulnerable to scan data artifacts. Our human body segmentation algorithm advances the state of the art to overcome the above limitations and we present experimental results obtained using both real and synthetic data that confirm the validity, effectiveness, and robustness of our approach.
\end{abstract}

Index Terms-Human body shape analysis, Reeb graph, scattered 3-D range data segmentation, 3-D surface topology, 3-D whole-body scanners.

\section{INTRODUCTION}

$\mathbf{T}$ HE PAST FEW years have witnessed the emergence of three-dimensional (3-D) imaging technology that enables full scanning of the human body (HB) surface with reasonable measurement accuracy as well as at an acceptable computational cost. This advance facilitates the exploitation of the HB form in various areas such as anthropometrical research [1]-[3], clothing design [4]-[6], and virtual human animation [7], [8]. Although the raw data generated by the HB scanner requires substantial main memory and storage resources, it contains little semantic information. To achieve effective and efficient use of body scan data, it is often necessary to partition the whole scan data set into subsets corresponding to the principal body parts.

Manuscript received June 21, 2004; revised February 9, 2005. This work was supported by the U.K. EPSRC under Grant RG 533801 and by the U.K. Imaging Faraday Partnership. This paper was recommended by Associate Editor D Goldgof.

$\mathrm{N}$. Werghi is with the College of Information Technology, Dubai University College, United Arab Emirates (e-mail: nwerghi@duc.ac.ae).

Y. Xiao and J. P. Siebert are with the Department of Computing Sciences, University of Glasgow, Glasgow, U.K. (e-mail: yjxiao@dcs.gla.ac.uk; psiebert@dcs.gla.ac.uk).

Digital Object Identifier 10.1109/TSMCB.2005.854503
This segmentation provides the basis for a high-level representation of the scan data and is a prerequisite for further semantic analysis. For example, in medical applications, the segmentation process provides an Atlas for extracting data belonging to limbs that can then be used to support further analysis such as fitting generic limb models. These models can then be used to automate specific clinical protocols, such as spinal curvature assessment. Applications dealing with the estimation of HB motion from range image sequences [9], can exploit scan data segmentation when initializing the parameters of a HB tracking algorithm. HB scan segmentation is also useful for online garment shopping applications [5], [6] as it can contribute toward providing accurate body measurements and sizing.

Many attempts to devise a framework for the segmentation of objects that are human-like in shape have been reported in the literature [10]-[13]. Most of this previous work is based on contour-based segmentation techniques whereby points of discontinuity in the range data are first detected and then dynamically grouped into contours using various techniques, such as energy-minimization, when processing deformable curves [14]. However, automatic segmentation of real HB data is a more challenging problem, firstly because the body shape is both articulated and deformable and secondly because the scan data is by nature nonuniformly sampled and may exhibit gaps and may be corrupted by noise. It was therefore necessary to explore new techniques in order to formulate approaches that would be better able to cope with these challenges. In his pioneering work, Nurre [15] approximated the body structure by a stick-template representing the head, the two arms, the two legs and the torso. His goal was to segment the body into six segments corresponding to these parts. This approach combines a global shape description, namely moments analysis, and local criteria of proximity which are derived from a priori knowledge of the relative positions of the body parts in the standard posture (standing body with arms held at the sides). The range data is organized into slices of data points. The horizontal slices are stacked vertically and the data points are assigned to the different body parts according to the slice's topology and its position in the body. While this work achieved considerable progress toward the automatic decomposition of HB scan data, it has been criticized for imposing the requirement to limit body poses to a strict standard posture and for its lack of robustness against noise, gaps in the data, and variations in shape and posture of the HB. There have been many subsequent attempts to improve Nurre's approach. For example, Decker et al. [16] improved the localization of the key landmarks of the HB by applying differential operations on slice shape attributes. Although a degree of improvement resulted, this 
approach could not remedy the limitations of Nurre's approach. Recently, Wang et al. [17] proposed a new approach based on a Fuzzy logic framework, however, this again was restricted to standing postures. Their segmentation technique involved local curvature analysis of the slices and operates on mesh data that has undergone several preprocessing stages. The overall performance of this approach remains identical to that of Nurre.

From the above it is evident that the approaches developed so far are restricted by their underlying assumptions, and none of these has been able to overcome the standard posture restriction. Furthermore, most of these approaches suffer from instability when applied within real applications that must cope with noisy and corrupted 3-D HB scan data. In addition, to date no evidence citing the repeatability of these previous algorithms has been reported in the literature. By definition, HB scan data segmentation must be of practical utility, it must be robust to variation in the body surface shape stemming from biological factors such as age, genetics, etc. It must also cope with changes of body posture as well as with the diversity of the scan data sources. While ad hoc techniques might work for special cases, they cannot address the above stringent requirements. In a recent publication [18], the authors presented an approach that successfully addressed some of the previously discussed issues. However this approach can only deal with moderate variations around the standard posture.

In this paper we propose a general topological analysis framework that offers a systematic way to segment HB body data. The salient feature of this framework is that it can cope with body shape variations, posture changes, rigid transformations and diverse sources of scan data. Furthermore, our approach does not require any pre-processing stages, operates on 3-D point-cloud data, and does not rely on local feature analysis, which would be vulnerable to deficiencies in the scan data.

The novel aspects of this paper are 1) the extension of the Reeb graph concept to sets of scattered data points, which represents an extension to the work of Biasotti et al. [19] which explores the use of Reeb graph applied to polygonal meshes; 2) a simple and efficient technique for computing the geodesic distance map of HB shape (and generally of a three-dimensional shape) with respect to a source point; 3 ) a robust technique, for constructing the discrete Reeb graph (DRG), which can cope effectively with data deficiencies; and 4) a new functional surface segmentation method based on the concept of the DRG applied to the HB surface.

The remainder of this paper is organized as follows. Section II describes the theoretical foundations of our approach, namely Morse theory, the Reeb graph and geodesic distance. Section III describes the implementation of the segmentation approach and the mechanisms involved. Section IV validates our proposed framework via experimental results. Finally, in Section V, we provide a summary of the main findings of this paper and make suggestions for future research.

\section{MORSE THEORY AND THE REEB GRAPH}

Morse theory can be thought of as generating the classical theory of critical points (maxima, minima and saddle points) of smooth functions on a smooth manifold. Specifically Morse theory states that for a generic function defined on a closed compact manifold, the nature of its critical points determines the topology of the manifold. Morse functions are generic functions whose critical points are nondegenerate (Hessian matrix of the function at the critical point is nonsingular). For a Morse function, the critical points determine the homology groups of the manifold, which in turn fully describe its topology. Moreover the way the manifold is embedded can be coded using a Reeb graph, as proposed by Reeb [20] to represent the evolution and arrangement of level-set curves on a manifold. A Reeb graph describes the configuration of and relationship between critical points and provides a way to understand the intrinsic topological structure of a shape. Morse theory and the Reeb graph have been used in many applications such as shape matching [21], shape coding [22]-[24], surface compression [25], volume visualization [26], terrain analysis [27] and 3-D skeletonization [28], [29]. The last publication cited is particularly close to our work. We will describe and compare in detail the related approaches in Section III-F.

A Reeb graph can be defined as follows.

Definition 1: Let $f$ be a real-valued function on a compact manifold $M$. The Reeb graph of $\boldsymbol{f}$ is the quotient space of the graph of $\boldsymbol{f}$ in $M$ by the equivalence relation " $\sim$ " defined by $\left(X_{1}, \boldsymbol{f}\left(X_{1}\right)\right) \sim\left(X_{2}, \boldsymbol{f}\left(X_{2}\right)\right)$ if $\boldsymbol{f}\left(X_{1}\right)=\boldsymbol{f}\left(X_{2}\right)$ and $X_{1}$ and $X_{2}$ are in the same connected component of $\boldsymbol{f}^{-1}\left(\left(\boldsymbol{f}\left(X_{1}\right)\right)\right.$.

Roughly speaking, the two pairs $\left(X_{1}, \boldsymbol{f}\left(X_{1}\right)\right)$ and $\left(X_{2}, \boldsymbol{f}\left(X_{2}\right)\right)$ are represented as the same element in the Reeb graph if the values of $\boldsymbol{f}$ are the same and if they belong to the same connected component of the inverse image of $\boldsymbol{f}\left(X_{1}\right)$ or $\boldsymbol{f}\left(X_{2}\right)$. Actually one element in the Reeb graph of a compact manifold represents all points having the same value under a real function. The Reeb graph is a representation of the evolution and arrangement of these groups of points, also called level-sets. Fig. 1(a) illustrates an example of a Reeb graph for a torus. The function $f$ is the "height" function which here simply returns the value of the coordinate $z$ of a point $X$. In the corresponding Reeb graph, a point in a branch represents isovalued and connected points on the manifold. From bottom to top, the level-sets on the torus expand, split, merge and then become smaller. The Reeb graph gives an intuitive description of the evolution of level-sets, where diamond points denote the level-sets passing through saddle points on the torus. By applying the Reeb graph to a HB, we get a tree-like representation as illustrated in Fig. 1(b). Extremal points lie on the head top, hand tips and the bottom of the feet. Saddle points are located at the armpits and groin. Moreover, the branches in the Reeb graph reflect the body parts of the human figure, i.e., arms, legs, torso, and head. Therefore, if we succeed in retrieving the level-sets in these branches, we can partition the input point-cloud data into sub-sets approximately corresponding to the body parts of the human shape.

\section{A. The Morse Function}

For standard postures such the one shown in Fig. 1(b), where the human figure stands in the measuring platform with arms held at the sides and legs separated, the simple height function $h(X)=z$ that returns the $\mathrm{z}$ coordinate of a point $X$, is an optimal choice because the orientations of the body parts in such 


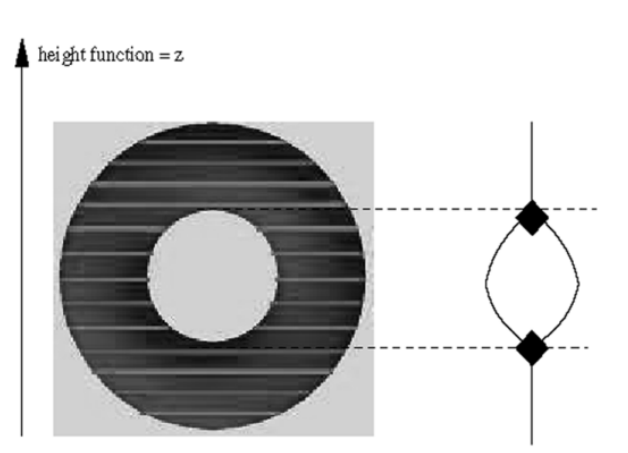

(a)

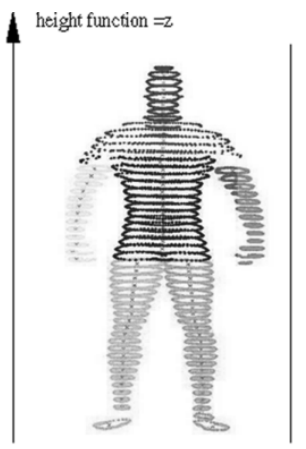

(b)

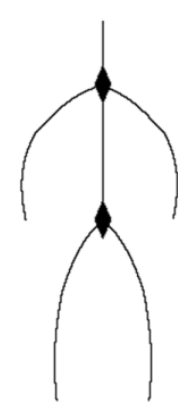

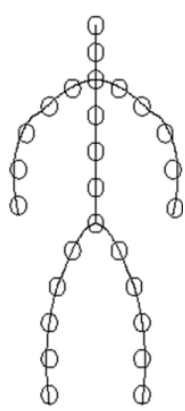

(c)

Fig. 1. (a) Reeb graph of a torus. (b) Reeb graph of a HB shape. (c) Discrete Reeb graph.

postures are orthogonal to the cross-sectional planes inferred by the height function. For nonstandard postures where the human figure is not constrained to be in a standing position, both the arms and legs as well as the whole body can have arbitrary orientations. The only constraint is that the body limbs must be separated from each other everywhere except at their joints. For such postures, the simple height function cannot guarantee a Reeb graph faithful to the $\mathrm{HB}$ anatomy. This limitation emanates from the fact that the height function is not invariant under rigid transformation or under deformation inferred by whole body transformation or body part movement. Therefore, to be capable of handling nonstandard postures, it is imperative that the Morse function be invariant under these transformations, i.e., a function that keeps the same value as long as the topology of the surface is preserved. The curvature function could be a candidate as it is invariant under rotation and only very slightly affected by body movements. However, it is very sensitive to noise and data deficiencies that would lead to highly unstable Reeb graph structures. Therefore, it is not appropriate for our application which must handle scattered noisy scan data that might be corrupted by many deficiencies such as holes, gaps and nonuniform sampling. To find a function that overcomes these problems, we employed the geodesic distance [31] defined as the length of the shortest path connecting two points. The geodesic distance is invariant to the rotations and transformations produced by body movements and is resistant to data corruption and perturbation. Thus the geodesic distance metric underpins a stable Reeb graph with respect to these aspects. The function defined as

$$
\rho_{S}(X)=\operatorname{gd}(X, S)
$$

where $\operatorname{gd}(X, S)$ returns the geodesic distance from a point $X$ to a source point $S$, is a reasonable candidate for a Morse function. However the location of the source point might affect, to a certain extent, the structure of the Reeb graph as will be shown later.

Another candidate for a Morse function is the function defined as

$$
\sigma(X)=\int_{Y \in \text { surface }} \operatorname{gd}(X, Y) d_{\text {surface }}
$$

This function represents the sum of the geodesic distances between the point $X$ and all the points on the body surface. In ad- dition to being resistant to geometric transformations and deformations produced by body movements, this function is not related to any source point and therefore guarantees a stable Reeb graph. Intuitively, the $\sigma$ function presents low values for points located at the center of the body (torso area), for which the distance to other points is relatively small, and high values for points located at the body extremities. On the other hand, this function is computationally expensive when compared to $\rho_{S}(x)$ as it will be described in Section III-A.

\section{B. The $D R G$}

Classical Morse theory is concerned with only nondegenerate critical points of smooth functions (Morse functions) on smooth manifolds. The notion of the Reeb graph in its standard form is defined with respect to smooth and continuous surfaces. There have been several successful attempts to extend the Reeb graph to discrete surfaces e.g., [19], but the surface data is required to be organized into polygonal meshes. In practice, our data format does not comply with this requirement, as it consists of an unorganized cloud of 3-D data points which have been corrupted by noise, gaps and nonuniform sampling. We present a Reeb-graph extraction technique that is compatible with this type of data. For clarification of terminology, hereafter we refer to Reeb graph extracted from such data as the DRG to distinguish it from the classical Reeb graph. The concept of the DRG is described in the following definitions.:

Definition 2 (Connectivity of Point Sets): Two point sets $P=$ $\left\{p_{i}\right\}, i=1 \cdots m$ and $Q=\left\{q_{j}\right\}, j=1 \ldots n$ are defined as connected if $\exists p_{i} \in P$ and $\exists q_{j} \in Q$ such that $\left|p_{i}-q_{j}\right| \leq \tau$.

$\left|p_{i}-q_{j}\right|$ denotes the distance between points $p_{i}$ and $q_{j}$ and $\tau$ is a given threshold. The above definition also holds for the connectedness between two points for the particular case where the sets $\mathrm{P}$ and $\mathrm{Q}$ contain a single point each.

Definition 3 (Connective Point Set): A point set $C$ is connective if $\forall$ subset $\Omega \subset C$ and $\Omega \neq \emptyset, \Omega$ and $\bar{\Omega}$ are connected. Here $\bar{\Omega}$ denotes the complement of $\Omega$ in C. Definition 3 defines a "tight" point set in which all the points are connected.

Definition 4 (Level-Set Curve): A level-set curve is an isovalued connective point set, that is a group of points, that share the same Morse function value, and which forms a connective point set.

Dentition 5: A Discrete Reeb graph is a non-oriented twodimensional (2-D) graph, where a node represents a level-set 
curve and where an edge represents a connection (in the sense of Definition 2) between two adjacent level-set curves.

Based on the above definitions, the construction of the DRG involves the following tasks.

Step 1: Establishing Level-Sets: Level-sets are groups $\mathcal{G}_{i}, i=1 \ldots M$ of iso-valued data points defined as $\mathcal{G}_{i}=\left\{X, f(X) \in\left[v_{i}, v_{i+1}\right]\right\}$, where $f$ is the Morse function and $v_{i}, i=1 \ldots M+1$ is a set of discrete equidistant values.

Step 2: Establishing Level-Set Curves: Each level-set is decomposed into a group of level-set curves, using the criteria outlined in Definition 3, and where the threshold $\tau$ is set to $d$ which stands for the minimum distance between a pair of points.

Step 3: Building the Connectivity Between Nodes: Two nodes in two adjacent level sets $\left(\mathcal{G}_{i}\right.$ and $\left.\mathcal{G}_{i+1}\right)$ are linked if their corresponding level-set curves are connected, according to Definition 2. However, the related threshold $\tau$ is set to $\beta d$, where $\beta$ is a parameter used to tune the precision of the connection.

By following the above steps, we can construct progressively a graph containing all the nodes and their associated links. Fig. 1(c) depicts a DRG of the HB shape. This graph has the appearance of a discrete version of the graph in Fig. 1(b), where the continuous branches are replaced with successively linked nodes. The DRG extends the concept of Reeb graph to discrete surfaces, and thus permits topological analysis of scan data.

\section{The SEgmentation}

The segmentation process involves three tasks, namely: 1) computing the Morse function; 2) extracting the level-sets; 3) decomposing these level-sets into connected level-set curves; and 4) extracting the different branches. Task 3 in essence comprises DRG construction. The implementation of these tasks within the segmentation process and the complexity of the overall algorithm depend on the adopted Morse function. When the simple height function is used (i.e., when dealing with standard postures), the four tasks are carried out within a single stage in one pass algorithm. When the $\rho_{S}$ function is used, tasks 1 and 2 are performed simultaneously. Alternatively, when the $\sigma$ function is employed the four tasks are executed consecutively. The following sections will shed light on these aspects.

\section{A. Computation of the Morse Function $\rho_{s}$ and $\sigma$}

Both Morse functions $\rho_{S}$ and $\sigma$ involve the computation of geodesic distances. In the literature, Dijkstra's algorithm [30] has been the most popular tool for computing geodesic distances between a group of points and a source point. In addition, it provides the path from any point to the source point. However, this algorithm implies a significant computational cost. Indeed, it requires that the group of points be organized in a graph, where a node is associated to each point and edge represents a connection between pair of points, according to a proximity criterion. The construction of such graph infers a computational complexity that can go up $O\left(N^{2}\right)$, where $N$ is the number of points. Dijkstra's algorithm itself, in its optimal implementation, infers a complexity of $O(E+N \log (N))$, where $E$ is the number of edges in the graph. For these reasons and because finding the paths to a source point is not required in our application, we preferred not to use this algorithm. Instead, we developed an efficient algorithm based on a wavefront propagation technique. It is based on the following principle: Given a wave centered on a manifold, then all the points on the wavefront have the same geodesic distance to the wave center and thus form a level-set. Our wavefront propagation algorithm operates on a binary voxel grid since it is easy to define a neighborhood in voxel space and to then traverse connected voxels. Due to these well-behaved properties, wavefront-propagation on a voxel grid can have a very simple mathematical form as follows:

$$
\left\{\begin{array}{l}
W_{0}=\left\{v_{s}\right\} \\
W_{i+1}=\left(W_{i} \oplus e-\left(W_{i} \oplus e\right) \cap S_{i}\right) \cap \bar{S}_{i}
\end{array}\right.
$$

where $W_{i}$ is the wavefront generated on the $i$ th iteration of the algorithm; $v_{s}$ is the source voxel; $S_{i}=\sum_{j=0}^{i} W_{j} . \bar{S}_{i}$ is the complement set of $S_{i} \oplus$ denotes the morphological dilation operator and $\mathbf{e}$ is a $3 \times 3 \times 3$ structuring element composed of 27 1 -valued voxels. The wavefront starting position is located at the source voxel associated with the source point and the wavefront then iteratively spreads on the voxelised surface from this location. In each iteration, the wavefront is the level-set containing voxels of the same geodesic distance to the source point. The attractive aspect of this technique is that it simultaneously extracts the level-sets while it computes the $\rho_{S}$ function. It is easy to prove that the computational complexity in each iteration is $O\left(n_{i}\right)$, where $n_{i}$ is the number of voxels in $W_{i}$. Therefore, the complexity of the whole algorithm is $O(N)$, where $N$ is the number of all 1-valued voxels. This linear complexity allows efficient calculation of geodesic distances and level-sets. Therefore the computation of the function $\rho_{S}$ is carried out by simply applying the above algorithm after selecting a source point. Fig. 2(a) illustrates wavefront-propagation applied on a simple ellipsoid surface. Row 1 in Fig. 2 depicts grey level mapping of the $\rho_{s}$ function, related to a posture instance, and corresponding to different locations of the source point, namely the head (column f), the torso(columns g, h, i, and $j$ ), the knee (column k), and the hand (column 1). The grey level varies from white (which corresponds to the minimum value, at the source point) to the black (largest value). Row 2 depicts the corresponding level-sets. We can observe that while the level-set orientations follow the body limbs in all cases, they do present some dissimilarities, particularly at the junction areas.

The computation of the $\sigma$ function is more complex as it involves for each data point the sum of geodesic distances from that point to all the points in the body surface. The discrete approximation of $\sigma$ in (2) is

$$
\sigma(X)=\sum_{i}^{N} \operatorname{gd}\left(X_{i}, X\right)
$$

Using (1), the above equation can be rewritten as $\sigma(X)=$ $\sum_{i}^{N} \rho_{X}\left(X_{i}\right)$. This indicates that the computation of $\sigma$ at a given point requires computing the function $\rho$ for all the 1 -valued voxels. Making thus the complexity of the whole algorithm that calculates the $\sigma$ function for all the points to be $O\left(N^{2}\right)$. 


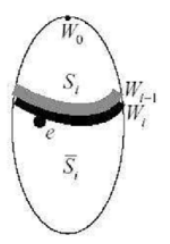

(a)

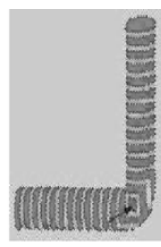

(b)

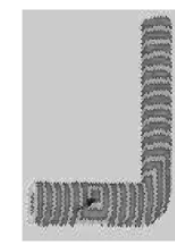

(c)

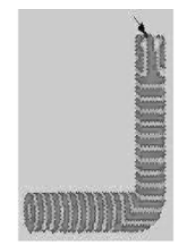

(d)

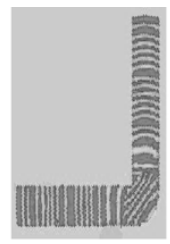

(e)
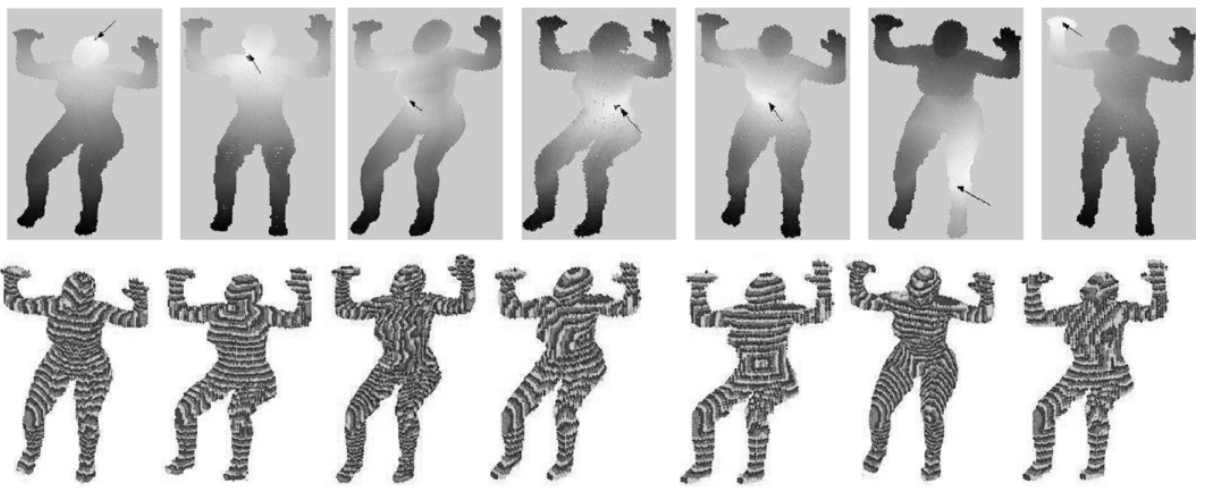

(2)

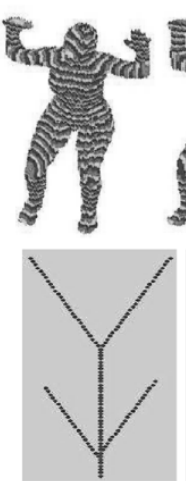

(f)

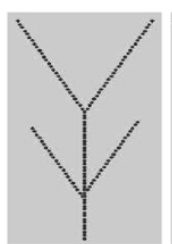

(g)

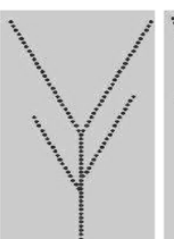

(h)

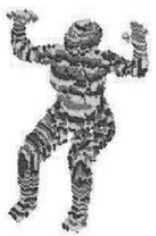

(b)

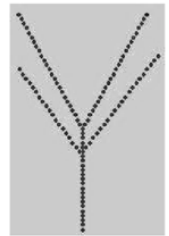

(c)

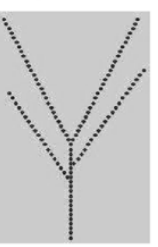

(i)

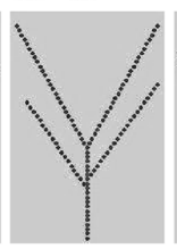

(j)

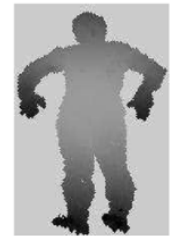

(d)

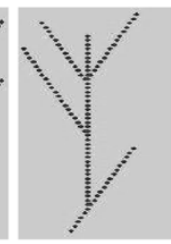

(k)

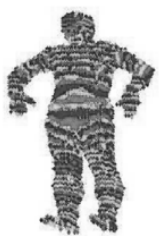

(e)

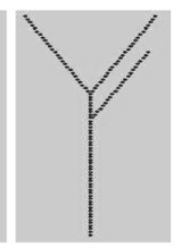

(1)

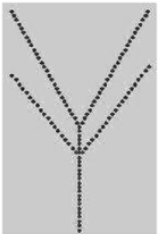

(f)

Fig. 2. (a): Wavefront propagation on a simple elliptic surface. (b), (c), (d) Level-sets of $\rho_{S}$ function, related to an L-Shaped object, and corresponding to source points located at the junction, the middle and the extremity of a branch respectively. (e) Level-sets of $\sigma$ function. Row 1: grey level mapping of the $\rho_{S}$ function for different source point locations (indicated by the arrows). Rows 2 and 3: corresponding level-sets' patterns and DRGs, respectively. Row 4: grey level mappings of the $\sigma$ function, the corresponding levels-sets' patterns and DRGs related to two different postures.

\section{B. Source Point Location and Its Effect on the Level-Sets and the $D R G$}

While the $\rho_{S}$ exhibits nice properties in terms of efficiency and ease of implementation, an effective deployment of this function for constructing the DRG, and for performing the separation between branches, depends to some extent on the location of the source point however. Ideally, the use of geodesic distance would permits us to extract level-sets that maintain a consistent orientation relative to the HB pose. Locally, this would result in the recovery of "slices" parallel to the principal axes of human limbs. Unfortunately, this desirable behavior is compromised at areas comprising junctions (and also within the neighborhood of the source point) and the patterns resulting from such corruption are dependent on the location of the source point itself. Fig. 2(b), (c), and (d) illustrates this effect on a simple L-shaped object, showing the different level-sets that are produced by the $\rho_{S}$ function corresponding to three different source points located at respectively: the junction area, the middle, and the extremity of one branch. By observing their corresponding patterns at junction areas clear dissimilarities become apparent.
The disparity between the level-set's behaviors in the three cases makes their decomposition into subsets, and the correspondence of these subsets to the their associated branches, unlikely to result in an identical separation between these branches. For the purpose of comparison, we show in Fig. 2(e) level-sets of the $\sigma$ function for the same object. We notice that the level-sets, seemingly behave like those related to the junction-located source point case. Indeed, both of them exhibit a degree of symmetry at the junction area.

Regarding the reconstruction of the DRG, ideally, we would like to have a DRG structure that is similar to the "standard" HB DRG depicted in Fig. 1(c). This structure reflects the anatomy of the HB shape, and thereby facilitates the identification of the branches. However, the stability of this structure is guaranteed only for source points selected at the central area of the body, that is the torso-head area. Fig. 2 (row 3 ) illustrates this aspect. It shows a group of DRGs obtained for different source point locations. We can observe that for cases where the source point is located within the torso-head area a DRG structure is generated which is close to the standard form displayed in Fig. 1(c). On the other hand, in those cases where the source point is lo- 


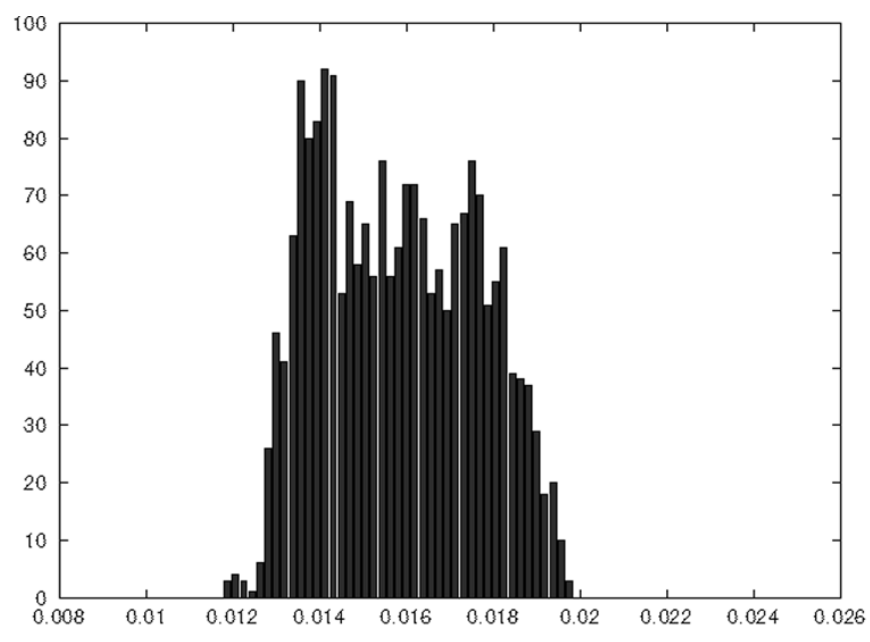

(a)

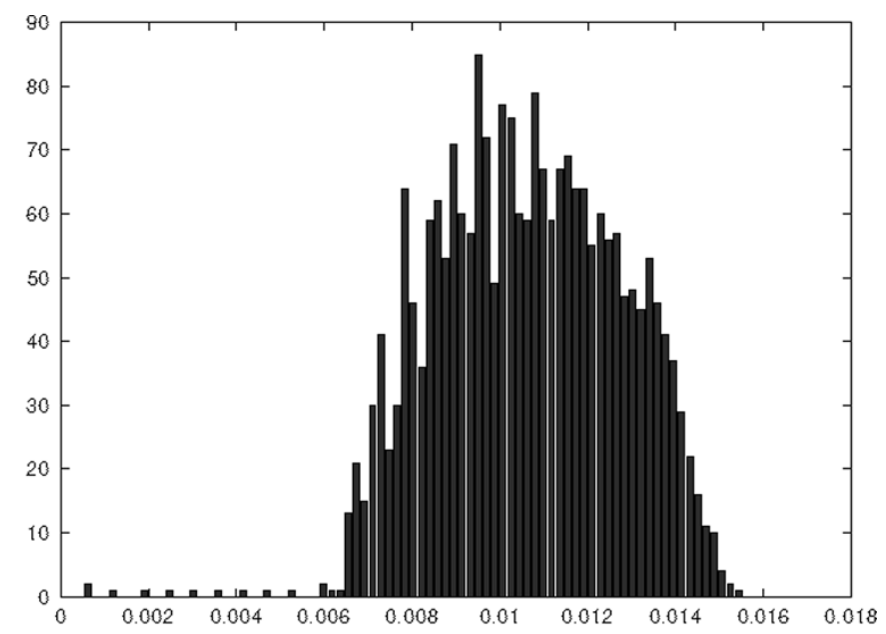

(b)

Fig. 3. Histogram of the distances between the most closest pairs of scan data points. (a) Cyberware scanner. (b) Wick \& Wilson scanner.

cated at the hand and the knee, the recovered DRG appears to have become corrupted. These observations suggest that in order to obtain a standard DRG the source point must be selected at the neighborhood of the medial axis of the body (i.e., the torso axis). While we do not have a theoretical basis for this hypothesis, we believe that it is linked to the fact that the satisfaction of that condition infers a symmetric distribution of the $\rho_{S}$ function with respect to the medial axis of the body. As the grey level mappings show, in Fig. 2(row1), cases exhibiting a symmetric distribution of the $\rho_{S}$ function induce a standard form of DRG, as opposed to cases where the symmetry of the $\rho_{S}$ function is severely violated (row 1 , columns $\mathrm{k}$ and $\mathrm{l}$, for source points located at the knee and the hand).

In contrast to the $\rho_{S}$ function, the $\sigma$ function which by definition has no dependance on a source point, exhibits a centered and a symmetric distribution (Fig. 2, row 4, a and d) from which it is possible to infer a stable DRG as shown in (Fig. 2, row 4, $\mathrm{c}$ and f). Therefore, when a $\rho_{S}$ function is employed, a suitable source point can then be obtained via manual selection or automatically using simple heuristic methods. One plausible method is the following: 1) compute the $\rho_{S}$ function for an arbitrary source point; 2) search for the point at which $\rho_{S}$ is closest to the average value; and 3) repeat 1) and 2) until the location of the source point converges. The point of convergence will be then located nearby the geodesic center of mass, in the torso-head area, and can therefore be selected as a suitable source point.

\section{Threshold Setting}

Setting the appropriate range of the threshold $d$ involved in step 2 and step 3 of the DRG construction should be performed with care since large values might introduce "short-circuit" edges in the DRG while small values can render the graph excessively sparse. In either case, the topological integrity of the HB shape might not be preserved. In our application, however, since the global geometry of the HB shape is known, some constraints can be used in the second case to eliminate false segments (some criteria related to this aspect are proposed in Section III-D1). Therefore we have to care only about the maximum value allowed for the threshold. For this purpose we used a practical approach which consists of first estimating the resolution $\epsilon$ of the scan data. This was conducted as follows. We determined the set of closest pairs of data points (i.e., nearest neighbor tuples) over a large area of the scanned data. Then we set the resolution to the weighted average deduced from the related distance histogram (the term distance here refers to the distance between the pairs of points). Fig. 3 shows two distance histograms corresponding to two portions of scan surface obtained from two HB scanners, namely a Cyberware scanner and a Wicks \& Wilson scanner (these scanners are discussed in Section IV). The corresponding resolutions are $\epsilon=1.6 \mathrm{~mm}$ and $\epsilon=1.1 \mathrm{~mm}$, respectively. The expression of the threshold can then be defined as $d=K \epsilon$, with the minimum value of $\mathrm{K}$ being set to 2 . This value would normally lead to the most precise segmentation, however at the expense of increased computational time. A larger value of $\mathrm{K}$, might reduce the accuracy of the segmentation, though our experiments (Section IV) showed that a reasonable topology-preserving segmentation can be obtained with up to $K=7$, as long as the the separation between the body parts is larger than the threshold $d$.

\section{DRG Construction}

The scan data is first organized into a voxel grid. The size of voxel is $d \times d \times d$ where $d$ is the threshold outlined in Step 2. When the height function is adopted (i.e., when dealing with standard postures), a group of iso-valued data points comprising a "slice" of points is obtained by intersecting a plane of a certain height with the body surface. When dealing with nonstandard postures, the $\rho_{S}$ or $\sigma$ is computed for each point in the voxel grid. For the $\rho_{S}$ function, the level-sets are implicitly extracted in this stage as described in Section III-A, whereas for the $\sigma$ function they are extracted subsequently. Each level-set of data points is then decomposed into level-set curves and the DRG is constructed according to Steps 2 and 3 described in Section II-B.

1) DRG Analysis and Branch Extraction: In this stage, the DRG is analyzed to detect critical nodes and to extract the branches corresponding to body parts. For the sake of clarity we shall describe the methodology for the case of a standard posture where the height function is employed. The general 


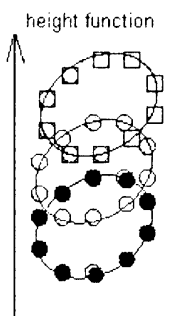

(a)

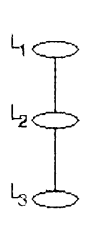

(b)

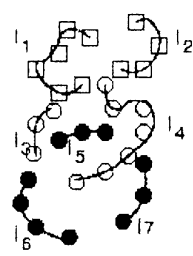

(c)

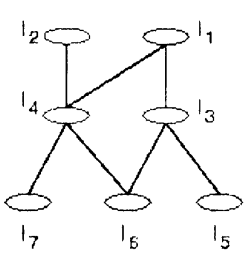

(d)

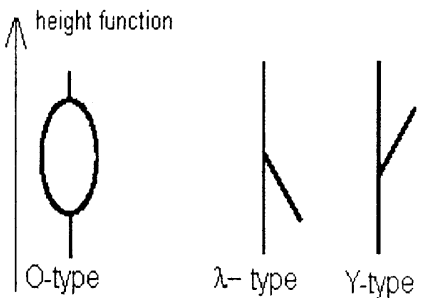

(e)

Fig. 4. (a) Three slices representing a portion of clean data. Each slice contains a single level-set curve (group of linked points), thus each slice is mapped into a single node in the DRG (b). (c) Data corruption causes each slice to decompose into several level-set curves. This results in a disorganized graph (d). In this example, the original nodes $L_{1}, L_{2}$, and $L_{3}$ have degenerated respectively into $\left(l_{1}, l_{2}\right),\left(l_{3}, l_{4}\right)$ and $\left(l_{5}, l_{6}, l_{7}\right)$. (e): Three primary patterns in DRG.

principles of the methodology remain valid for the other Morse functions and we shall point out any dissimilarities where these arise.

While it appears to be straightforward to detect the critical nodes in a DRG related to clean and well organized scan data [such as that depicted in Fig. 1(c)], this task is not trivial for real scan data. The data deficiencies (noise, holes and gaps) produce topological disturbances that lead to "false" critical nodes. This results in a corrupted tree structure as illustrated in Fig. 4. For simplicity, the example shown in this figure covers only a portion of three adjacent slices. In "clean" data, each slice consists of an organized set of connected points 4(a). Thus, each slice represents a single level-set curve. This will result in a DRG composed of three nodes 4(b). For real data, a slice might be composed of several level-set curves because of data corruption, leading thus to several nodes per slice. The example in Fig. 4(c) shows three level-set curves for slice 1 and two level-set curves for each of slice 2 and slice 3 . The three initial nodes $L_{1}, L_{2}, L_{3}$ have degenerated into seven nodes $l_{1}, l_{2}, \ldots, l_{7}$. Setting the connections amongst the nodes as the final step of the algorithm (as described in step 3, Section II-B) leads to a disorganized graph [4(d)] that results, at the scale of the whole scan, in the DRG degenerating into a chaotic graph. The challenge therefore is to be able to recover the topological structure of the measured HB from such a corrupted graph. This problem is tackled as follows: Firstly, the nodes in the DRG are arranged level-bylevel, and a link can only exist between two nodes in adjacent levels. This property cannot be damaged by the decomposition of nodes. Secondly, we have identified three primary topological configurations that appear in the DRG and have termed these as O-type, $\lambda$-type and Y-type patterns respectively [Fig. 4(e)]. For example, the group of nodes $\left(l_{7}, l_{4}, l_{2}, l_{1}\right),\left(l_{6}, l_{4}, l_{1}, l_{3}\right)$, and $\left(l_{6}, l_{5}, l_{3}, l_{1}\right)$ represent a Y-type, an O-type and a $\lambda$-type, respectively. O-type patterns comprise two saddle nodes connected by two branches and this pattern reflects data anomalies (wholes, gaps) because the topology of the human form cannot produce such a topological configuration. Indeed, this argument also applies to Y-type patterns since a standard posture cannot generate a Y-type configuration. Therefore, O-t and Y-type occurrences in the DRG originate only as a result of deficient data. The $\lambda$-type and Y-type patterns are topologically identical however, since each comprises one saddle node and one leaf branch. Therefore, in order to distinguish between them, we introduce topographic information, namely, the direction of the height function, to allow a down/up ( $\lambda$-type/Y-type) leaf branch cate- gorization. Given the preceding, if O-types and Y-type patterns occur in the DRG, we know that they are caused by data corruption. In order to distinguish genuine $\lambda$-type patterns from false ones that have been produced by data deficiencies, we assume that the size of a leaf branch associated to a true $\lambda$-type pattern must not be smaller than the size of the smallest body part. This assumption is reasonable since the scan data is unlikely to contain gaps or holes at the scale of the body limbs, as such cases can be easily prevented by some form of quality control during the data acquisition phase.

Based on these considerations, the following criteria are used to identify "true" $\lambda$-type patterns which comprises "true" branches and saddle nodes: 1) a 'true' branch is downward and must satisfy: $\mid h_{\max }($ branch $)-h_{\min }($ branch $) \mid>h_{\text {lim }}$ where $h_{\max } / h_{\min }$ (branch) denotes the maximum/minimum value of a branch in the height direction, and $h_{\mathrm{lim}}$ is a threshold that represents the minimum length allowed for a branch and 2) a "true" saddle node has at least two branches.

The strategy followed to reject false critical nodes and identify 'true' branches for nonstandard postures remains the same except that the categories of patterns in the DRG will be reduced to only two types, namely the O-type (caused by data noise) and either the $\lambda$-type or the Y-type, depending on which orientation the level sets are constructed. We choose to construct the level-sets in the orientation of the increasing geodesic distances, thus allowing only O-type and $Y$-type patterns in the DRG. Therefore, the branch orientation in criterion 1 is amended to upward and the Morse function is set to the $\rho_{S}$ function or $\sigma$ function depending on which one is adopted. Criterion 2 remains unchanged, however.

\section{E. The Segmentation Algorithm}

The very constrained configuration of the HB in standard-postures and the simplicity of the height function in terms of computation permit the simultaneously extraction of level-sets with the construction and analysis of the DRG to extract the branches. The segmentation algorithm, named here Algorithm 1 (shown below), contains only a one-pass search from the bottom to the top of the scan data. In this pass, the critical nodes representing the bottom of the feet, groin, hand tips, armpits and head top are detected, and the 'true' branches between these critical nodes are extracted. The identification of branches corresponding to the body parts then becomes very simple, whereby the branches between the groin and bottom of the feet correspond to legs and the branches between armpits and hand tips correspond to the 
arms. The reminder of the data corresponds to torso and head. For nonstandard postures, the Morse function and the level-sets are first computed (in one stage for the $\rho_{S}$ function and in two stages for the $\sigma$ function) and thereafter, Algorithm 1 is applied (after setting the 'true' orientation of the branches to up) to extract the critical nodes and the true branches.

\section{Algorithm 1: Notation \\ Slice: A level-set \\ Node: A level-set curve in a slice. \\ Class: A group of connected nodes. \\ Class(Node): The Class containing the Node. \\ Branch: A 'true' branch connected to a 'true' saddle node.}

$f$ : The Morse function (height function for standard postures) and $\rho_{S}$ function or $\sigma$ function for nonstandard postures

Code:

For each slice

Group data points into level-set curves

For each level-set curve

Assign a node to this level-set curve

If it is the 1-st slice

$$
\text { Class (node) }:=\{\text { this node }\}
$$

Else

Extract the group of nodes $\left(l_{1}, l_{2}, \ldots, l_{m}\right)$, connected to node, from the previous slice

If $m=0$

Class(node) $:=\{$ this node $\}$

Else

classes verifying:

From $\left\{\operatorname{Class}\left(l_{i}\right), i=1,2, \ldots, m\right\}$ select the

$$
\begin{aligned}
& \left\{C_{j}:\left|f_{\max }\left(C_{j}\right)-f_{\min }\left(C_{j}\right)\right|>h_{\mathrm{lim}}, j=1,2, \ldots, n,(n \leq m)\right\} \\
& \quad \text { If } n \geq 2 \\
& \quad \text { For each } C_{j} \\
& \quad \operatorname{Branch}_{k}=C_{j}, \quad k:=k+1
\end{aligned}
$$

\section{End For}

End If

$\operatorname{Class}($ node $)=\{\operatorname{Class}($ node $\left.)\} \bigcup\left\{\operatorname{Class}\left(l_{1}\right)\right\} \bigcup \cdots \bigcup\left\{\operatorname{Class}\left(l_{m}\right)\right\}\right\}$

\section{End If}

\section{End If}

End for

\section{End for}

\section{F. Summary of the Approach and Comparison With 3-D Skeletonization}

The HB scan segmentation approach involves four tasks and the implementation depends on the adopted Morse function. Table I summarizes the different versions. Of the different tasks,
TABLE I

STAGES AND TASKS INVOLVED IN THE DIFFERENT VERSIONS OF THE SEGMENTATION PROCESS. THE First COLUMN INDICATES THE EMPLOYED MORSE FunCtion. THE SECOND COLUMN LISTS THE NuMBER OF INFERRED Stages. THE THIRd COlumn Indicates the TASKS INVOlved IN EACH StaGe

\begin{tabular}{c|c|l}
\hline Morse function & Stages & Tasks \\
\hline \hline height & 1 & $\begin{array}{l}\text { Level-sets extraction, DRG Construction } \\
\text { and branch extraction }\end{array}$ \\
\hline \multirow{4}{*}{$\rho_{S}$} & 1 & Computing $\rho_{S}$ and level-sets extraction \\
\cline { 2 - 3 } & 2 & DRG construction \\
\cline { 2 - 3 } & 3 & Branch extraction \\
\hline \multirow{5}{*}{$\sigma$} & 1 & Computing $\sigma$ \\
\cline { 2 - 3 } & 2 & level-sets extraction \\
\cline { 2 - 3 } & 3 & DRG construction \\
\cline { 2 - 3 } & 4 & Branch extraction \\
\hline
\end{tabular}

the computation of the Morse function is the most time consuming. The height function implies the simplest implementation, because it does not infer any computation. This permits us to perform all of the tasks in a single stage. Conversely, the $\sigma$ function requires the sequential execution of the four tasks, and represents the most costly implementation $\left(O\left(N^{2}\right)\right)$. The computational time related to this function is of the order of several hours on a Pentium IV, $1.7 \mathrm{Ghz}$ computer. The $\rho_{S}$ function, exhibits a reasonably tolerable computational cost. It exhibits an overall complexity of $\mathrm{O}(\mathrm{N})$. Despite the fact that the $\sigma$ infers a stable DRG, we prefer the $\rho_{S}$ function, because of the considerable disparity in terms of computational cost. Furthermore, our experiments showed that the two versions are similar in performance. The presented algorithm operates on a voxel grid and the number of voxels containing data points is small compared with the number of raw data points in the scan. However, it is straightforward to recover segmented raw HB scan data points from the segmented voxel data structure.

The approach of Verroust and Lazarus [29], mentioned previously in this paper, is the closest to ours in terms of theoretical background. Although both approaches involve similar concepts, namely, geodesic distance and level-sets, there are several fundamental differences, namely the objective of our task and the complexity and robustness of our respective implementations. In the following section, we shall detail these aspects, while emphasizing the characteristics of our approach.

Firstly, regarding the task undertaken, the two approaches target different objectives, namely skeleton construction and functional-based segmentation. Regarding implementation, the skeletonization process in [29] involves five stages: 1) establishing a neighborhood graph, where nodes represent data points, and an edge between a pair of nodes represents a connection between the corresponding pair of points, according to the m-nearest points rule; 2) computing a geodesic graph out of the neighborhood graph-this graph is actually a tree composed of geodesic paths joining the data points to a source point. The Dijkstra algorithm is used to compute the geodesic paths as well as the geodesic distances between the data points and the source point; 3) extracting level-sets of isovalued points using the geodesic graph; 4) Partitioning each level-set into subsets corresponding to the different branches of the surface; and 5) computing the centroids of connected subsets in each level-set and joining them, via the geodesic graph, to construct the skeletal curves. The approach was implemented 
on powerful Indy machines. In this process the extraction of the level-sets goes through the construction of a neighborhood graph and the use of Dijkstra's algorithm. This results in a high computational complexity. Indeed, although the neighborhood graph algorithm is optimized, its complexity is estimated to be $O\left(n k^{2}\right)$, where $n$ is the number of data points and $k$ is the average of the number of points contained in the neighborhood of each point. The value of $k$ is not small given the potentially very large number of points in the raw scan data. Dijkstra's algorithm has a complexity of $O(e+n \log (n))$ where $e$ is the number of edges in the neighborhood graph. Proceeding as in [29], to determine the level-sets in our application, will induce an intolerable computational cost. Based on the preceding implementation, we estimate that to process one HB containing 13000 points would require approximately $20 \mathrm{~h}$ to execute on a Pentium IV, 1.7-GHz computer. In the contrary, the $\rho_{S}$ version of our approach where the extraction of the level-sets infers a complexity of $\mathrm{O}(\mathrm{N}), \mathrm{N}$ being the number of 1-valued voxels, takes around $70 \mathrm{~s}$ to achieve the segmentation of the whole scan. Yet the most important feature of our work with respect to [29] is its robustness. Indeed, it is not clear how the approach in [29] can cope with an irregular sampling distribution of data points, data corruption and deficiencies. Since in order to obtain a valid skeleton, faithful to the body anatomy, each obtained subset (stage 4 of the skeletonization process, mentioned above) must correspond to a branch and thereby: 1) contain a single connected component and 2) form a closed curve. The non satisfaction of condition 1 , results in false branches in the skeleton. The authors in [29] showed an instance of this case related to a body scan, presenting false branches, at the level of the feet, caused by the irregular distribution of the data points and the presence of gaps. The violation of condition 2, causes the centroid to be shifted (stage 5) from its actual location at the branch axis implying thus a distorted curve. The resulting skeleton will not then reflect correctly the actual body template. Cases such as those depicted in Fig. 9(b) and (c) for instance, cannot be accommodated by the approach in [29]. Actually, the authors in [29] recognized that the validity of their approach is conditional upon having data points that are regularly distributed on the object surface. Our approach, on the contrary, copes rather effectively with different types of data corruption due to the mechanisms implemented in the DRG analysis that eliminate false branches while preserving correct connectivity. Regarding segmentation accuracy, it appears that operating on raw data points and the use of Dijkstra's algorithm as in [29] improves segmentation accuracy because this approach is able to extend the computation of the $\rho_{S}$ to the interior of the edges of the geodesic graph by interpolating the endpoint values of the edge. This in turn permits a finer mapping of the $\rho_{S}$ function. While this result appears to be attractive, as it allows the possibility of further refining the level-sets and improving the accuracy of the segmentation, it is also very likely that this benefit will be cancelled by the corruption of the level-sets at the junction areas of the body (see Section III-B). It must also be noted that this limitation applies equally to our approach. Nevertheless the established thresholds, namely $d=K \epsilon$, and $\beta d$, involved respectively in setting connections between pair of points and level-set curves, permit us to control to a limited extent the accuracy of the segmentation in our approach.

\section{EXPERIMENTS}

A series of experiments were conducted using real and synthetic data to test the validity of our approach. The performance and the robustness of the corresponding algorithms were assessed with respect to: 1) variation of the HB shape; 2) variation of scan source; 3 ) severe corruption of the scan data; and 4) variation of the HB posture.

Real-world HB scan data was collected from different sources, namely the Cyberware website [32], the CAESAR project website [3] and the HB scanner located within EdVEC, Edinburgh Virtual Reality Centre [34]. The scans of the first two sources were acquired by Cyberware whole body scanners WB4 [32]. This scanner, uses laser-based technology in which a laser beam is projected on the body. The beam profile is captured by different cameras around the body and 3-D points are then extracted from each camera view to be combined into a single 3-D point cloud representing the body surface. The acquisition time of this scanner is approximately $17 \mathrm{~s}$. The set of collected scans contains 25 scans of different individuals including males and females (only 4 are shown in this paper). Each scan contains of the order of 13000 data points. The third source is a Wicks \& Wilson HB scanner [33], which is based on a Moire fringing technology where fringe patterns, projected onto the body surface, are captured by four cameras, with a moving mirror system providing another four viewing positions. The sets of 3-D points extracted from each camera (triangulating with the fringe projector) are combined together to form the whole scan. The scanning time of this device is around $8 \mathrm{~s}$, yet the person is required to stand very still during the scanning. Furthermore, the space inside the scanner is very limited and allows little freedom of body movement. For these reasons, it was difficult to perform scans for nonstandard postures. However, we did manage to obtain a few nonstandard postures and collected a set of ten scans (seven are shown in the paper) related to three different male individuals. The number of points in each scan is approximately 11000 points. Synthetic HB scans were obtained using POSER software [35]. The data sets were generated by randomly sampling the surface of four different models (two men and two women, two with clothes and two without). 16 simulated scans were then extracted representing human figures in different non standard postures. First, we conducted preliminary tests in order to: 1) compare the performance of the algorithm versions related to the $\rho_{S}$ and $\sigma$ Morse functions and 2) assess the appropriateness of the threshold selection with respect to $d=K \epsilon$ (described in Section III-C) and $\beta d$ used to establish connections between adjacent level-set curves. Fig. 5 shows trials made with two postures, using the $\rho_{S}$ version (a) and the $\sigma$ version (b) and we can observe that the segmentation results are not significantly different. Threshold setting $d=K \epsilon$ was investigated out as follows: We segmented a HB scan (in standard posture) several times, each with a different threshold $d=K \epsilon$, with $\mathrm{K}$ varying from 2 to 7 . These tests were carried out with the algorithm version that utilizes a height function. Fig. 6 (Rows and 1 and 


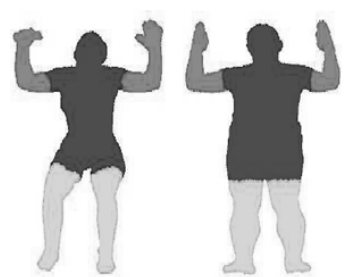

(a)

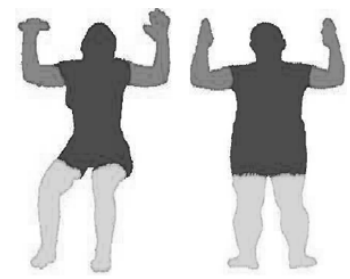

(b)
Fig. 5. Cyberware scan and a Wick \& Wilson scan segmented using respectively a $\rho_{S}$-based version (a) and a $\sigma$-based version (b) of the approach.

(1)
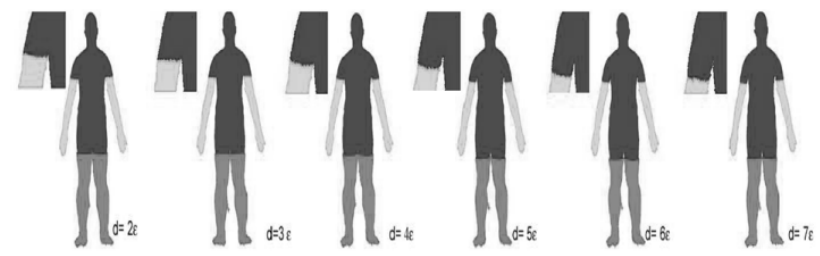

(2)
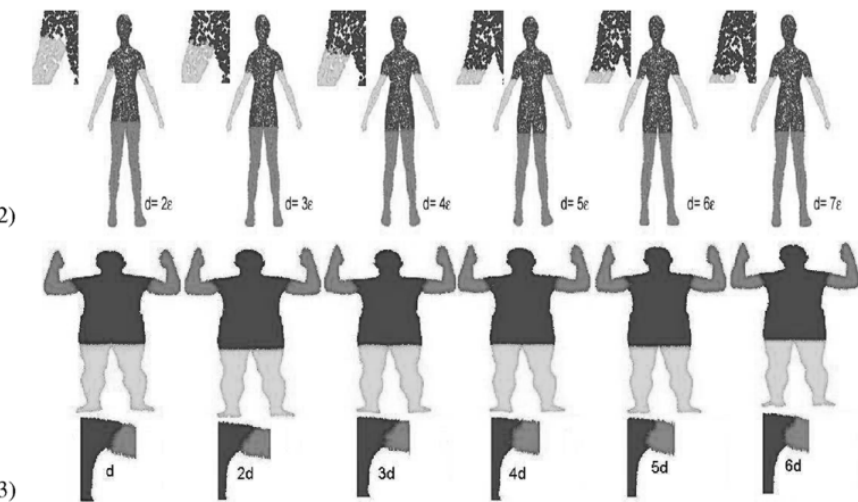

Fig. 6. Rows 1 and 2: A Wick \& Wilson HB scan and Cyberware scan segmented using a sequence of six thresholds ranging from $d=2 \epsilon$ to $d=7 \epsilon$, where $\epsilon$ is the scanner resolution estimated in Section III-C. Row 3: A Wick $\&$ Wilson HB scan segmentation results corresponding to six thresholds $\beta d, \beta=1, \ldots, 6, d=2 \epsilon$.

2) shows that the overall segmentation appears to be similar throughout the trial. However, by examining the zoomed areas around the armpit we can observe a decrease in the segmentation accuracy as the threshold becomes larger (a decrease in segmentation accuracy is clearly visible for $K \geq 4$ ). This behavior is caused by "short-circuit" edges between nodes of the arm and nodes of the torso. These short-cuts occur when the distance separating the arm and the torso becomes relatively small compared to the threshold $d$. Threshold setting $\beta d$ was investigated as follows. We set the threshold $d$ to the best precision $(2 \epsilon)$ and we segmented a nonstandard posture six times, each with a different value of $\beta$ varying from 1 to 6 . The segmentation was carried out with the $\rho_{S}$-based version of the approach. The results are depicted in Fig. 6 (row 3). The observation of the zoomed area around the right armpit, across the different trials reveals a slight improvement in the accuracy, as $\beta$ increases until it reaches $3-D$. Beyond that value it appears to stabilize.

Next, we applied the segmentation algorithm to a collection of scans obtained from different sources, including real and synthetic data. Because of space constraints we show principally the results related to nonstandard postures. Exhaustive results corresponding to standard postures have been published in [18].

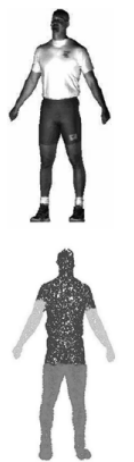

1
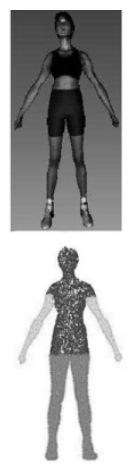

2
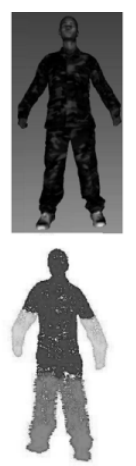

3
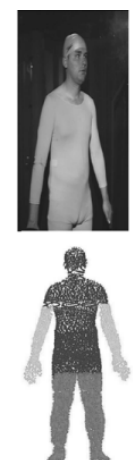

4
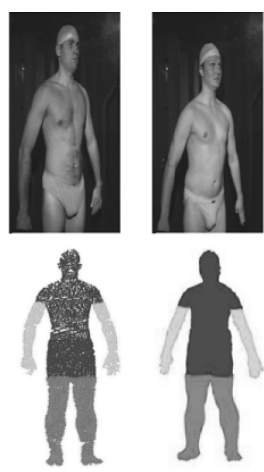

6
Fig. 7. Segmentation of HB scans acquired with the Cyberware scanner (1, 2, 3 ) and Wicks \& Wilson scanner $(4,5,6)$.

Fig. 7 depicts images of the scanned persons, in a standard posture taken during the scanning process and the corresponding segmented scans. These scans were acquired with the Cybeware scanner $(1,2,3)$ and the Wicks \& Wilson scanner $(4,5,6)$. We can observe that the scans are segmented with reasonable accuracy. The scan 3 in Fig. 7 corresponds to that of a fully dressed person. The corresponding surface presents many irregularities caused by wrinkles, in comparison to other scans which present smooth surfaces. Despite the surface irregularity, the segmentation result is reasonable and confirms that the algorithm can cope with ill-conditioned (non smooth) surfaces.

For nonstandard postures, validation experiments were performed using the second version of the algorithm, that involves the Morse function $\rho_{S}$. A first series of tests were performed on HB scans, related to people in a seated posture, and collected from the CAESAR website [3]. The Results are depicted in Fig. 8(a). We can see that all the data sets are clearly segmented into five subsets corresponding to the arms, legs and the torso, despite the sitting posture of the subjects and the variety of body shapes. A second series of tests was conducted over several scans acquired from Wick \& Wilson scanner [33], the results are depicted in Fig. 8(b), which also shows images of the scanned persons. The results reveal reasonable segmentations that produce correctly separated body parts.

Because of the current shortage of real scans of nonstandard postures we had to resort to computer-simulated data sets in order to examine additional postures. A first set of artificial postures was obtained as follows: We conformed a parameterized HB template to a Cyberware HB scan and then animated the conformed model by varying the template parameters. Some instances from this set are shown after segmentation in Fig. 8(c). The results illustrate correct segmentation for all the samples. The second set was obtained by generating 16 synthetic scan models in different postures using Poser software [35]. These data were obtained by randomly sampling the animated mesh models, thus generating scattered data point sets of similar properties to real scan data. The scans were extracted from four different models comprising a man and a woman, in both dressed and undressed states. The dressed models included the hair. Fig. 8(d) and (e) shows the 16 scans and the segmentation results. We can see that all the data sets are clearly decomposed into the five principal parts for all the different postures, illustrating the robustness of the algorithm with respect to rigid transformation and deformation. Fig. 8(e) 

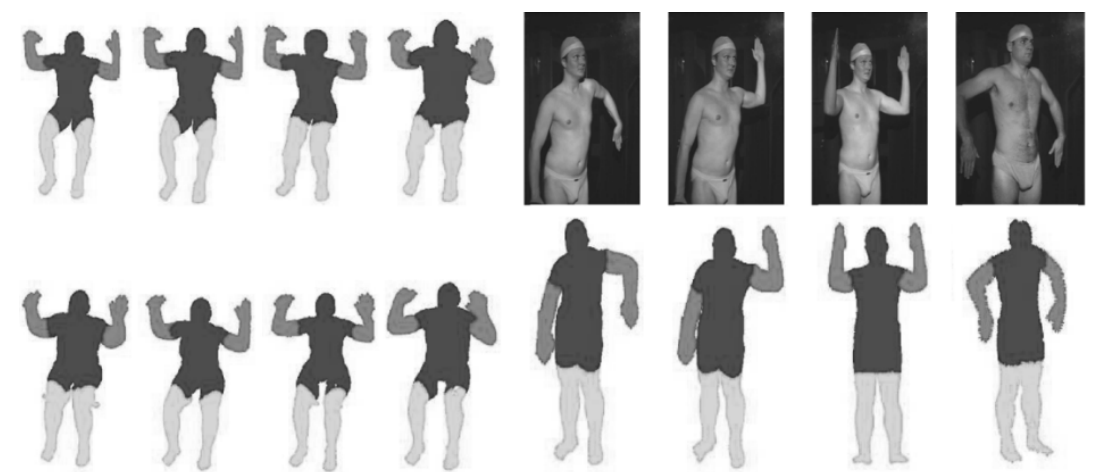

(a)
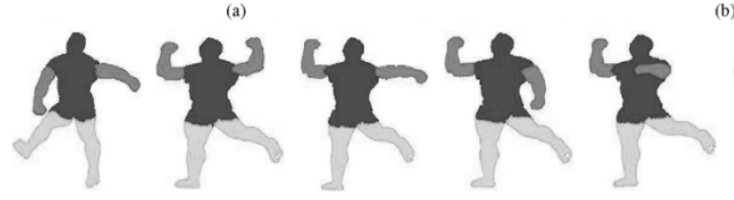

(b)

(c)
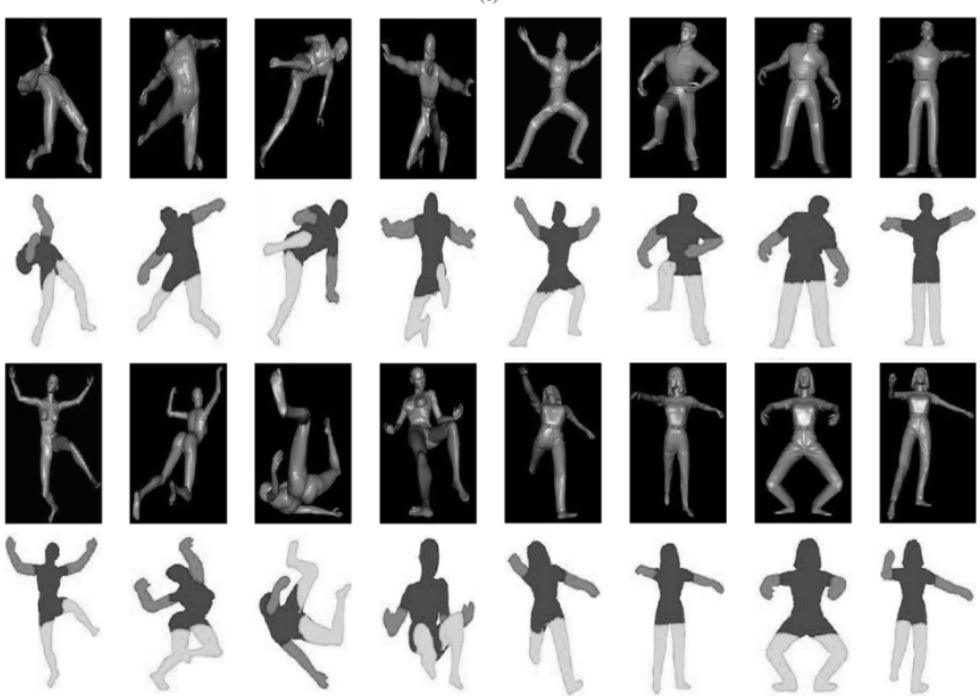

(d)

(e)

Fig. 8. (a) Segmented real scans corresponding to setting postures acquired withe the Cyberware scanner. (b) Segmented Wick \& Wilson scans related to nonstandard postures. (c) Segmentation results of an animated Cyberware scan. (d) and (e) Segmentation results of simulated scans corresponding respectively to scans of naked and dressed characters.

in particular, illustrates the robustness of the algorithm to false branches. Indeed, if we examine for instance, the head of the dressed woman, we can observe wrinkles and waves characterizing the hair surface. These features are likely to cause false critical nodes that consequently generate false branches. However, our algorithm did not fail when applied to this data set. Also we can observe that fingers are not segmented separately, even though some of them appear to be partially detached from the hand and therefore disposed to generate true branches in the DRG. This is explained by the fact that the first criterion established in Section III-D1 states that a true branch must have a length above a given threshold, which is set here to the size of smallest body part, namely the arm. Therefore branches inferred by body parts smaller than the arm, such as the fingers and the head will be discarded by the algorithm.

\section{A. Robustness With Respect to Data Deficiencies}

It is also worth mentioning that these results were obtained with poorly scanned data as illustrated in Fig. 9(a), which shows a zoomed area around the groin. The nonregular sampling of the data and the presence of gaps and holes are clearly visible. To further test the robustness of the segmentation algorithm, we corrupted the data by creating large holes in a group of scans representing a variety of postures. The results presented in Fig. 9(b) and (c) reveal a consistent segmentation over all of the corrupted scans: all of these scans are properly decomposed into the five body parts, despite the presence of large gaps. These results confirm that the algorithm is capable of discarding effectively the O-type critical nodes described in Section III-D1. In another experiment we corrupted the scan data by adding Gaussian noise of different amplitudes to the data points. A representative set of the associated segmented scans are depicted in Fig. 10. These validation trials illustrate, therefore, the robustness of our approach against noise disturbance and severe data deficiencies.

\section{CONCLUSIONS}

This paper presents a topological framework for the segmentation of HB scans. The framework extends the Reeb graph con- 


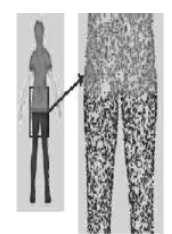

(a)

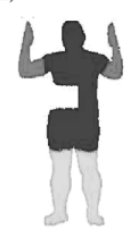

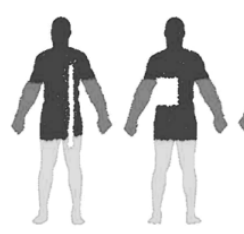

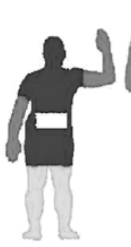

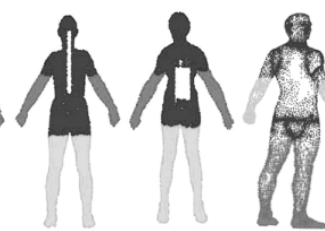

(b)

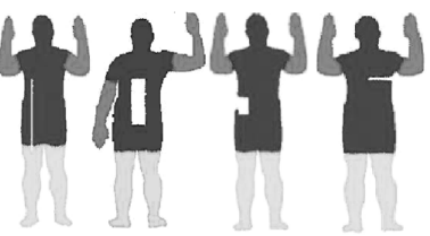

(c)

Fig. 9. (a) Zoomed image illustrating the distribution of the scan data. (b), (c) Segmented HB scans corrupted by large holes.
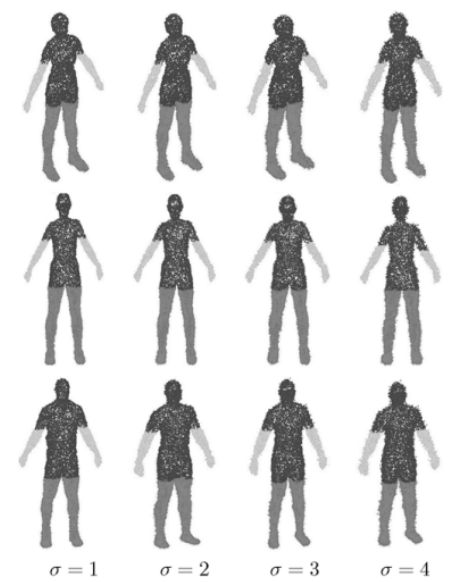

Fig. 10. Segmented HB scans corrupted with a Gaussian noise of different amplitudes.

cept to unorganized clouds of data points by defining connectivity notions. Compared to work previously reported in th eliterature describing on HB segmentation, our framework is differentiated by the following features: 1) it handles directly the raw scan data without the need of any preprocessing or pre-formatting stages; 2) it involves only topology-based techniques; 3 ) it can cope with arbitrary postures; 4) it offers different configurations in order to accommodate different categories of $\mathrm{HB}$ posture; and 5) no post-processing stage is required. With respect to other 3-D skeletonization frameworks, our approach is distinguished by the following aspects: 1) intrinsic robustness, that allows it to cope with data deficiencies and severe corruptions; 2) an efficient implementation; and 3) it provides a solution to the problem of locating the source point, by using a source-point independent Morse function, although at the expense of additional computational cost.

The scan sets used in the experiments were collected from different sources and cover a rich variety of HB shapes and profiles including some severely damaged scans. The results confirm the robustness of the approach with respect to the diversity of scan sources, diversity of the body shapes, rigid transformations, irregular distribution, and corruption of the scan data.

From a quantitative point of view, the experiments confirm the reliability and the repeatability of the two algorithms. In the 152 segmentation trials (58 are shown in this paper), the algo- rithms always segment the scan into five connected and compact parts that respect the topology of the HB. Cases of severe under-segmentation, over-segmentation, or cases where a segmented part contains disjoint subparts (e.g., from the arm and the leg), never occurred.

While the results show overall reasonable segmentations, it would appear to be difficult to achieve what would be considered a "perfect" segmentation to a human observer because of the ill conditioned behavior of the level-sets at the junctions areas. In fact, we believe that to achieve human segmentation performance would require techniques that go beyond our middlelevel processing approach. Nevertheless, the segmentation provided by our approach could be used as a starting point for finer processing and analysis. One might also ask to what extent the joint areas detected by our approach can be used to locate actual useful body landmarks, which are used for instance in garment sizing. While our approach is suitable for delimiting the locale of such landmarks, detecting their precise location may be literally beyond the grasp of purely scan-based approaches, since these landmarks often correspond to bony points below the skin, which are usually detected and located by palpation. There is some hope that a 2-D/3-D approach to landmark detection based on combining both 2-D image features and 3-D surface features used in conjunction with explicit statistical knowledge, as encapsulated in an active appearance model, may provide a route to a more complete analysis of the human form. To this end, we propose that the techniques presented here could provide a useful means of constraining the search space by providing an initial annotation of the body.

Being based on topological analysis, our framework is intrinsically not qualified to handle postures where limbs are joined together, for example closed legs, or arms touching the torso. Dealing with such cases requires that the contours of discontinuities between joined parts of the body be detected and labeled, using local surface analysis and differential geometry techniques or explicit model fitting. The work developed in [12], for example, could perhaps afford an appropriate framework for handling the above cases.

It is quite plausible that our framework can be extended to deal with other variety of 3-D shapes. The approach should integrate mechanisms to accommodate with change of scale and shape. Some directions that can be explored are: 1) using multiple source points for the Morse function $\rho_{S}$, analyzing the resulting DRGs, and establishing criteria to reject those degenerate graphs; and 2) a fast implementation of the $\sigma$ function as it infers a stable DRG; in addition it can be adapted for a multiscale segmentation approach, in the absence of any prior knowledge about the object size.

\section{ACKNOWLEDGMENT}

The authors would like to thank Cyberware and the Caeser project, from which we obtained HB scans, and the Edinburgh Virtual Environment Centre for providing access to their HB scanning facilities. Finally, they would like to thank the UK Engineering and Physical Sciences Research Council who supported this work and also the reviewers for their constructive feedback. 


\section{REFERENCES}

[1] P. R. M. Jones and M. Rioux, "Three dimensional surface anthropometry: Applications to human body," Opt. Lasers Eng., vol. 28, no. 2, pp. 89-17, 1997.

[2] E. Paquet, K. M. Robinette, and M. Rioux, "Management of three-dimensional and anthropometric databases: Alexandria and Cleopatra," J. Electron. Imag., vol. 9, pp. 421-431, 2000.

[3] The Civilian American and European Surface Anthropometry Resource Project Website (2005). [Online]. Available: http://www.sae.org/technicalcommittees/caesar.htm

[4] R. P. Pargas, N. J. Staples, and J. S. Davis, "Automatic measurement extraction for apparel from a three-dimensional body scan," Opt. Lasers Eng., vol. 28, no. 2, pp. 157-172, 1997.

[5] D. Protopsaltou et al., "A body and garment creation method for an internet-based virtual fitting room," in Advances in Modeling, Animation, and Rendering, J. Vince and R. Earnshaw, Eds. Berlin, Germany: Springer-Verlag., 2003, pp. 105-122.

[6] F. Cordier, H. Seo, and N. Magnenat-Thalmann, "Made-to-measure technologies for an online clothing store," Comput. Graph. Applicat., pp. 38-48, Jan.-Feb. 2003.

[7] J. Starck and A. Hilton, "Human shape estimation in a multi-camera studio," in Proc. British Machine Vision Conf., Manchester, U.K., 2001, pp. 573-583.

[8] J. Starck, G. Collins, R. Smith, A. Hilton, and J. Illingworth, "Animated statues," J. Mach. Vis. Applicat., pp. 248-259, 2003.

[9] M. Lin, "Tracking articulated objects in real-time image sequence," in Proc. Int. Conf. Computer Vision, Corfu, Greece, 1999, pp. 648-653.

[10] D. L. Borges and R. B. Fisher, "Segmentation of 3-D articulated objects by dynamic grouping of discontinuities," in Proc. British Machine Vision Conf., Surrey, U.K., 1993, pp. 279-287.

[11] D. Dion Jr., D. Laurendeau, and R. Bergevin, "Generalized cylinders extraction in a range image," in Proc. IEEE. Int. Conf. Recent Advances in 3-D Digital Imaging and Modeling, Ottawa, ON, Canada, 1997, pp. 296-299.

[12] F. Ferrie, J. Lagarde, and P. Whaite, "Darboux frames, snakes and superquadrics: Geometry from the bottom up," IEEE Trans. Pattern Anal. Mach. Intell., vol. 15, no. 8, pp. 771-784, 1993.

[13] E. Trucco, "Inferring convex subparts from slice data," Patt. Recognit. Lett., vol. 1, no. 12, pp. 707-715, 1991.

[14] M. Kass and D. Terzopoulos, "SNAKES: Active contour models," Int. J. Comput. Vis., vol. 1, no. 4, pp. 321-332, 1988

[15] J. H. Nurre, "Locating landmarks on human body scan data," in Proc. IEEE Int. Conf. 3-D Digital Imaging and Modeling, Ottawa, ON, Canada, 1997, pp. 289-295.

[16] L. Dekker, I. Douros, B. F. Buxton, and P. Treleaven, "Building symbolic information for 3-D human body modeling from range data," in Proc. IEEE Int. Conf. 3-D Digital Imaging and Modeling, Ottawa, ON, Canada, 1999, pp. 388-397.

[17] C. L. Wang, T. K. Chang, and M. Yuen, "From laser-scanned to feature human model: A system based on fuzzy logic concepet," Comput.-Aided Des., vol. 35, no. 3, pp. 241-253, 2003.

[18] Y. Xiao, P. Siebert, and N. Werghi, "A discrete reeb graph for the segmemtation of human body scans," in Proc. IEEE Int. Conf. 3-D Digital Imaging and Modeling, Alberta, Canada, 2003, pp. 378-385.

[19] S. Biasotti, B. Falcidieno, and M. Spagnuolo, "Extended reeb graphs for surface understanding and classification," in Proc. Int. Conf. Discrete Geometry for Computer Imagery, Uppsala, Sweden, Dec. 2000, pp. 185-197.

[20] G. Reeb, "Sur les points singuliers d'une forme de Pfaff completement integrable ou d'une fonction numrique," Comptes Rendus Acad. Sciences Paris, vol. 222, pp. 847-849, 1946.

[21] M. Hilaga, Y. Shinagawa, T. Kohmura, and T. Kunii, "Topology matching for fully automatic similarity estimation of 3-D shape," in Proc. SIGGRAPH 2001, New York, 2001, pp. 203-212.

[22] C. Tai, Y. Shinagawa, and T. Kunii, "A Reeb graph-based representation for nonsequential construction of topologically valid shapes," Comput. \& Graph., vol. 22, no. 2, pp. 255-268, 1998.

[23] J. C. Hart, "Morse theory for implicit surface modeling," in Mathematical Visulaization, H. C. Edge and K. Plothier, Eds. Heidelberg, Germany: Springer-Verlag, 1998, pp. 257-268.

[24] Y. Shinagawa, T. L. Kunii, and Y. L. Kergosien, "Surface coding based on morse theory," IEEE Trans. Comput. Graph. Applicat., vol. 11, no. 5, pp. 66-78, 1991.

[25] S. Biasotti, M. Mortara, and M. Spagnuolo, "Surface compression and reconstruction using Reeb graphs and shape analysis," in Proc. Spring Conf. Computer Graphics, Bratislava, Slovakia, 2000, pp. 175-184.
[26] I. Fujishiro, T. Azuma, and Y. Takeshima, "Automating transfer function design for comprehensible volume rendering based on 3-D field topology analysis," in Proc. IEEE Conf. Visualization, San Francisco, CA, 1999, pp. 467-470.

[27] M. van Kreveld, R. van Oostrum, C. Bajaj, V. Pascucci, and D. Schikore "Contour trees and small seed sets for isosurface traversal," in Proc. Symp. Computational Geometry, Nice, France, 1997, pp. 212-220.

[28] M. Mortara and G. Patané, "Affine-invariant skeleton of 3-D-shapes," in Proc. Int. Conf. Shape Modeling and Applications, Banff, AB, Canada, 2002, pp. 245-252.

[29] A. Verroust and F. Lazarus, "Extracting skeletal curves from 3-D scattered data," Vis. Comput., vol. 16, no. 1, pp. 15-25, 2000.

[30] T. H. Cormen, C. E. Leiserson, and R. L. Rivest, Introduction to Algorithms. New York: McGraw-Hill, 1990.

[31] J. S. B. Mitchell, D. M. Mount, and C. H. Paradimitriou, "The discrete geodesic problem," SIAM J. Comput., vol. 16, no. 4, pp. 647-668, 1987.

[32] Cyberware Website (2005). [Online]. Available: http:www.cyberware.com

[33] Wicks and Wilson Website (2005). [Online]. Available: http://www.wwl.co.uk/wwl2/index.html

[34] Edinburgh Virtual Reality Center Website (2005). [Online]. Available: http://www.edvec.ed.ac.uk

[35] Poser Website (2005). [Online]. Available: http://www.curiouslabs.com

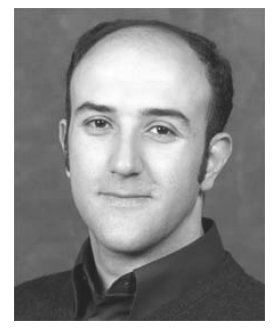

Naoufel Werghi (M'97) received the Ph.D. degree in computer vision from the University of Strasbourg, Strasbourg, France, in 1996, and the M.Sc. degree in instrumentation and control for computer vision from the University of Rouen, Rouen, France, in 1993.

He has been a Research Fellow at the Division of Informatics, University of Edinburgh, Edinburgh, U.K., and a Lecturer at the Department of Computer Sciences, University of Glasgow, Glasgow, U.K. He has also been a Visiting Professor at the Department of Computer and Electrical Engineering, University of Louisville, Louisville, KY. Currently, he is an Assistant Professor at the College of Information Technology, Dubai University College, UAE. His research interests are in the areas of computer vision and computer graphics, in particular 2-D/3-D image processing, 3-D shape analysis and modeling.

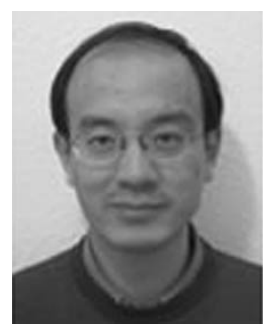

Yijun Xiao is currently a Research Assistant in the Department of Computing Science, University of Glasgow, Glasgow, U.K. His research interests are in the areas of computer vision, graphics, and image analysis, with particular focus on 3-D. He has authored or co-authored 14 international journal/conference papers.

Dr. Xiao is a member of the British Computing Society.

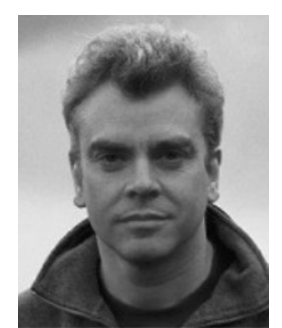

Jan Paul Siebert (M'01) received the B.Sc. and Ph.D. degrees from the Department of Electronics and Electrical Engineering, University of Glasgow, Glasgow, U.K., in 1979 and 1985, respectively.

$\mathrm{He}$ is currently a Senior Research Fellow in the Department of Computing Science, University of Glasgow, and the Group Leader for the Computer Vision and Graphics Laboratory. From 1991 to 1997, he was with the Turing Institute, Glasgow, developing photogrammetry-based 3-D imaging systems for clinical applications, and served as Chief Executive since 1994. Prior to this, he was a Scientist at BBN Laboratories, Edinburgh, U.K., from 1988 to 1991. His research interests include 3-D imaging systems and tools for human and animal surface anatomy assessment, and also robot vision systems based on biologically motivated principles. $\mathrm{He}$ has co-authored more than 60 international journal and conference papers in these areas. 